WellBeing International

WBI Studies Repository

1985

\title{
Applications of Laboratory Technology in the Evaluation of the Risk of Rabies Transmissions by Biting Dogs and Cats
}

\author{
Donald C. Blenden \\ University of Missouri \\ Manuel J. Torres-Anjel \\ University of Missouri \\ F. T. Satalowich \\ University of Missouri
}

Follow this and additional works at: https://www.wellbeingintlstudiesrepository.org/acwp_vsm

Part of the Animal Studies Commons, Other Animal Sciences Commons, and the Veterinary Preventive Medicine, Epidemiology, and Public Health Commons

\section{Recommended Citation}

Blenden, D.C., Torres-Anjel, M.J., \& Satalowich, F.T. (1985). Applications of laboratory technology in the evaluation of the risk of rabies transmissions by biting dogs and cats. In M.W. Fox \& L.D. Mickley (Eds.), Advances in animal welfare science $1985 / 86$ (pp. 221-246). Washington, DC: The Humane Society of the United States.

This material is brought to you for free and open access by WellBeing International. It has been accepted for inclusion by an authorized administrator of the WBI Studies Repository. For more information, please contact wbisr-info@wellbeingintl.org.

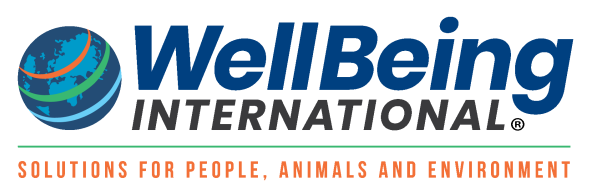




\section{APPLICATIONS OF LABORATORY TECHNOLOGY INTHE EVALUATION OFTHE RISK OF RABIES TRANSMISSIONS BY BITING DOGS AND CATS}

\section{Donald C. Blenden \\ Manuel J. Torres-Anjel and F.T. Satalowich}

Epidemiology and Public Health

College of Veterinary Medicine

University of Missouri

Columbia, Missouri 65211

\section{Introduction}

While rabies is not a common disease in domestic animal species of the United States, potential exposures to rabies in the form of bites are very common and increasing. A nationwide study conducted among general hospitals shows that 1 percent of emergency room visits are for animal bites, of which 80-90 percent are inflicted by the dog (Callaham 1980). This figure is conservative, as the study did not include pediatric hospitals, the bite of victims that progress only to a physician's office, or those that receive no medical care at all. In Missouri alone, this study would infer about 1500 dog bites per year reaching only the general hospital. The number of dog and other animal bites across the country is unknown but may safely be assumed to be staggering in magnitude.

The risk of acquiring rabies from an animal bite is one of the important considerations in medical treatment. While a significant number of the many bites are inflicted by the dog, the Center for Disease Control (CDC) reports an approximate figure of only 153 confirmed cases of rabies in the dog for 1982 across the United States. This figure is down from a high of about 250 in 1980 (U.S. Department of Health and Human Services 1983). This means that there is an inordinant difference 
between the number of bites and the number of cases of dog rabies; but, who at the time of a bite incident, can determine which biting animal is the one carrying rabies? The bite victim is one of a staggering yearly number; the biting animal is probably one of the large number which bites, but does not carry rabies. But, which is which? At the time of the bite incident, decisions must be faced concerning whether or not to treat the bitten person against rabies, and although all necessary information is often not available, the decisions must be made and cannot wait. The same principle of assessment applies to an animal which is bitten by a potentially rabid dog or cat, although the end consequences differ somewhat.

A scientifically accurate method of determining the rabies risk presented by the bite of an animal is to examine its brain tissue by immunofluorescence microscopy, sometimes supplemented by the inoculation of brain tissue into white mice. Properly treated and examined, the tissue containing rabies virus will fluoresce when examined under an ultraviolet microscope and brain tissue not containing rabies virus will not fluoresce; mice inoculated with tissue containing rabies virus will develop rabies, and those inoculated with virus-free tissue will not develop rabies. Brain examination has been a time-honored way of determining whether an animal that bites is infected with rabies in order to determine the risk of the bite to the person or animal bitten. The accuracy of brain examination via immunofluorescence microscopy approaches 100 percent both in sensitivity and specificity.*

An alternative to this drastic procedure is to confine the biting dog or cat for ten days and immediately obtain a reliable diagnosis of any illness present at the time of the bite, or any which develops within the period of confinement. This procedure is based on experimental observations conducted in the early 1960's. These observations ascertained that the dog or cat that is incubating rabies may have virus in its saliva for a maximum of three to four days prior to the development of the first symptoms of the disease (Vaughn et al. 1963, 1965). Thus, the healthy-appearing dog or cat that produces a rabies-dangerous bite and is confined will almost certainly be sick with rabies within the ten-day period. If it does not develop illness during the period, it can be assumed that the bite was not a rabies exposure. While a few exceptions to this rule have subsequently been described (lowering slightly the sensivitity), they are regarded as rarities and changes in bite management for these rarities is not warranted. It should be stressed that a confinement procedure is acceptable only for management of bites inflicted by the dog and cat; our knowledge of rabies in other species is inadequate to allow us to expand this procedure to include them. Indeed, there is

*A detailed discussion of sensitivity and specificity of tests follows. 
considerable reason to believe that other species (especially wild animal species) present an entirely different and increased risk. Thus, the confinement procedure for the dog and cat is another testing procedure approaching 100 percent in sensitivity and specificity.

A last alternative in the management of animal bites is to do nothing with respect to the potential of rabies exposure. While this is often the desire of the person responsible for the actions of the offending dog or cat, it can well be seen that the person or animal bitten is thereby placed into a category of much higher risk of contracting rabies from the bite. When bites are particularly serious and the risk of rabies is high, the bitten person is often started on antirabies treatment pending results of the confinement period. When the confined animal reaches the ten-day period in perfect health (with respect to rabies) it then becomes obvious that the initiation of treatment was unnecessary and can be stopped; but the risk in waiting to start is too high.

Today's alternatives in the handling of bites by the dog and cat are thus limited to those which either kill the animal, confine the animal (producing expense and a waiting period) or do nothing, the latter placing the person bitten at greater risk. All alternatives are characterized by great anxiety and emotional stress on the part of all parties involved. It is natural and necessary that serious bites are associated with increased pressures to conduct brain examination rather than confine the animal for ten days.

Until about twenty-five years ago, the dog was a principle reservoir of rabies in the United States, and the risk of dog bites transmitting rabies was consequently very high; indeed, the dog is still a principle reservoir worldwide. The main reservoir of rabies in the United States today has become the wildlife species, specifically the skunk, racoon, and several species of bat. The cat remains important as a biting species, and although these animals were not historically considered a significant reservoir of rabies, this situation is changing. The CDC reports that in 1981, the number of confirmed cases of rabies in cats (275) surpassed those in dogs (225) for the first time since 1975. Although the confirmed cases of rabies in both species have fallen in the ensuing years, the cat still leads the dog.

One attempt to improve the handling of bite cases has been accomplished in this twenty-five year period. That is the concept of "rabies-free areas," in which cases of rabies have not occurred in carnivores for a long period of time, and therefore the bites of carnivores carry greatly reduced risk of transmitting rabies (Marr and Beck 1976). Here, the difference between "very low" risk and "no" risk becomes important. Seldom can it be said that the rabies risk of a bite is absolutely zero; but it can be said that the risk of the bite transmitting rabies might be comparable to or lower than the risk of taking antirabies treatment, which, after all, is also very low, but not zero. 
Improvements in the medical management of animal bites to prevent rabies have occured because of the improvement of vaccines used for humans, not in the prevention of the need for the vaccine. While the vaccines are infinitely improved, they are still expensive, not totally risk-free, and tend to escalate the anxiety of a bite situation rather than producing a pallative effect as they are a defensive tool.

Concurrent with the decrease of rabies in the dog, the importance of the dog as a biting species has increased greatly. As a result, today we have the situation wherein the dog is much more likely to inflict bites, and is less likely to transmit rabies, but the technology of evaluation of the risk of rabies from bites has remained essentially the same. We still decapitate, however humanely, large numbers of dogs and cats in order to examine their brain tissue. Again, this is scientifically adequate or even ideal technology, but seems to be a grossly exaggerated and insensitive response, when it is clear that the rabies risk has greatly diminished. It is easily determined after the fact which death was necessary and which was not, but there are large numbers of dogs and cats sacrificed annually in order to find the relative few that present the risk of rabies. It seems logical that the application of modern technology can markedly lessen the need for brain examination; such technology is now available.

Following the idea that the treatment response often seems to be exaggerated in regards to animal bites of humans, we have conducted research with an overriding goal to lessen the need for the killing (and perhaps even some confinement) of animals for bite evaluation. Towards this goal, we hope to develop or apply existing technology in order to lessen the need for this killing and to contribute to the alleviation of the tremendous emotional distress produced in persons with real or imagined exposure to rabies or those who suffer the loss of a valued animal in order to conduct an examination. Making widely available the new and existing technology so it can be employed in the routine assessment of animal bites can lend supplemental, objectively obtained evidence to the body of information used to develop judgements regarding the relative risk of $\mathrm{dog}$ and cat bites. While the application of laboratory methods can improve evaluations, it is no panacea. The methodology will not answer all questions, and will not save much money, but it should allow more accurate assessments to be made and eliminate the killing of many animals. It will also reduce the number of antirabies treatments considered to be necessary, and grossly decrease the anxiety levels which so often commonly accompany bites. The employment of these new methods which depart from traditions and the confidence which is conferred by upon them by time and usage, requires that one differentiate between, and act on, the basis of degrees of risk. It is also essential that the desirability of preserving, rather than destroying, animal lives when appropriate be adopted as a goal. 
Today, the philosophy of brain examination most frequently is to justify why it should not occur. By adopting a positive attitude and employing laboratory methods to substantiate the opinions involved, the question then can become how brain examination is justified in each instance. To be perfectly clear, this discussion centers upon dog and cat bites only and applies to no other species. Indeed, realistically, brain examinations will not be eliminated entirely in the foreseeable future for any species, but the recent advances in technological knowledge should, in the long term, benefit both society and dogs and cats.

In addition to overcoming tradition, new technologies may encounter legal or regulatory obstacles. For example, many communities have ordinances requiring confinement and observation of biting dogs and cats for a period of ten days. Other ordinances require annual immunization of dogs and cats, but do not allow for the utilization of improved vaccines that produce two or three years duration of immunity. Such ordinances are slowly being replaced with those recognizing appropriate technological advancement. As our knowledge about rabies in wild animals indicates that the disease behaves differently in these cases, it is the sad reality that these bite incidences must still be handled in the traditional way. Considerable research must be done to determine the feasibility of new diagnostic technology when applied to wildlife, since laboratory assessment of the living wild animal may not answer the necessary questions, thereby endangering human life.

\section{How Are Bites Handled Today?}

\section{Situation A: A dog* bites a person and promptly escapes, never to be seen again. What should be done?}

The dog is gone, so no testing on it is possible. Unless the dog was accurately identified and its history known, the animal is a "stray." The only way to evaluate the probability of an exposure to rabies is for an experienced advisor to reconstruct the circumstances of the bite as accurately as possible. Any and all characteristics of the animal such as its species, its behavior or signs of illness at the time of the bite, evidence of provocation, any suggestion that the animal was known in the neighborhood to be a chronic "biter," knowledge of its vaccination status, the level of rabies infection in the community or many other factors which may help to assess the situation must be evaluated. Some situations can then be logically decided to be low in risk, others may be decided to be high risk ("low" risk can only rarely be interpreted to mean "no" risk). Most persons can decide for themselves on the level of risk they

\footnotetext{
*While the dog is used as an example, the discussion also applies to the cat.
} 
wish to undertake if they are presented with factual and objective understanding of the situation. The decision to treat or not treat with antirabies globulin and vaccine then becomes a bit more automatic.

Unfortunately, expert advisory services on animal disease and behavior are not always easily available or even less commonly consulted. These services should be made more widely available (many state health departments do not employ a veterinarian) and used to avoid unnecessary treatments, risk, and expense. The expert advisor should be a physician and/or veterinarian who is experienced and knowledgeable of the subtleties of rabies. Many persons are treated unnecessarily (Schnurrenberger et al. 1969) because physicians do not have access to the needed advice concerning the animal, and the risk of not treating is perceived as too great.

Situation B: A dog bites a person, is clearly sick or acting abnormally, and is confined and available for examination.

The illness of the animal should be evaluated by a veterinarian as soon as possible. If the animal has a diagnoseable illness other than rabies, the chance of a concurrent infection with rabies is remote. If the animal was hit by a car or some such incident, one must question: why was it hit? It could have been hit because of inability to react properly due to illness, perhaps rabies. Unless the illness is diagnosed with great certainty, it must be assumed to be rabies until proven otherwise; undiagnosed paralytic or neurologic disease carries high risk. This means either confinement to see how the illness progresses (rabies will usually progress and worsen within two or three days) or immediate laboratory examination, or both. "Laboratory examination" today means an examination of the brain for evidence of rabies virus. If a veterinarian is not available to examine the animal, the risk of erroneous diagnosis increases.

Situation C: A healthy-appearing dog bites a person, is captured, and is known to be a friendly type of dog.

The aggressive behavior of the animal must be interpreted as normal (if the animal is mistreated, injured, otherwise stressed or threatened) or abnormal (abnormal behavior is an early sign of rabies). The interpretation of the behavior must be done by an experienced person and the "provoked" or "unprovoked" bite must be viewed as through the eyes of the dog or cat, not the person evaluating. As an example, an animal will bite because of invasion of what it regards as its territory, which will have nothing at all to do with its owner's property line. So, the unprovoked bite (abnormal behavior) must be regarded as caused by rabies until proven otherwise. The bite because of provocation can be considered normal behavior, and so the risk is considerably lower. However, animals can carry rabies virus in the saliva before they begin to show symptoms 
(three to four days in the dog and cat). Therefore, the provoked bite of the healthy animal can transmit rabies, although the relative risk of this is much lower. The animal delivering a provoked bite, while presenting less risk of transmitting rabies virus, should be confined for ten days. At the first sign of illness during that period, immediate diagnosis and (often) brain examination is needed.

The unprovoked bite is considered a rabies risk until proven otherwise, as the bite is then regarded to be a symptom of illness, and immediate brain examination is needed. In a mild bite situation, confinement and observation is sometimes appropriate but in a serious bite situation, the risk increases and decisive action is essential.

Situation D: A healthy-appearing dog bites a person, is captured, and is known to be an aggressive dog or habitual biter.

The aggressive behavior can be regarded as normal, and that behavior can be expected to be magnified if the animal is provoked. The risk of rabies transmission from a normally aggressive animal is perhaps less to the person bitten than a bite by a normally placid dog. By the same token, the aggressive dog is more likely to inflict more serious bites, which increases the risk of rabies infection if the virus should happen to be present. Even though the bite likely reflects normal behavior, there is still the risk of the animal carrying rabies virus the few days before it shows symptoms. This dog or cat should be confined for ten days and/or brain examined immediately. If confinement is elected, an immediate diagnosis and perhaps brain examination is mandatory if illness develops.

It is the bites of these two latter categories of healthy-appearing dogs that encompasses the majority of bites (excluding those where the animal escapes), and also have lower risk of rabies transmission than the bites of sick animals. From these groups there are tremendous numbers of animals that are (necessarily) confined and/or brainexamined. There is much room for subjective interpretations of these situations: what is abnormal versus normal, provoked versus unprovoked, healthy versus unhealthy, mild exposure risk (to the person bitten) versus serious exposure to rabies, and so on? We desperately need a better way to supplement and make objective determinations to reduce needless confinements and killings, while not sacrificing the safety of the persons bitten and exposed.

Consider also the following. The circumstances considered in determining whether or not a bite was provoked are almost always viewed by only a few persons, and those persons are often subject to tremendous influence of observer bias; e.g., the child who is unable or unwilling to accurately recount the event, or the defensive and devoted owner whose dog "can inflict no wrong." In either case, or the myriad of variations on the same theme, the description of the event is relayed less than objec- 
tively. This information is then recounted to another party, often a medical person, always with the best of intentions but often with high levels of anxiety. Thus, it is up to the medical professional to assure that adequate information is elicited, and to proceed with appropriate decisions with the best information that is available. Although only 2-30 percent of dog bites in urban areas are attributed to ownerless animals (Beck 1981), perhaps as high as 50 percent of bites in rural areas are from animals that cannot be identified or captured for observation. These animals present a dangerous situation, as they have a greater likelihood of having been exposed to wildlife vectors of rabies virus. Therefore, the history of the animal is often the only resource available in these cases upon which to base treatment decisions. The obviously sick animal presents a well appreciated and easily interpreted risk. The real problem situations commonly boil down to questions of relative risk of the healthy biting dog or cat being subclinically infected with rabies but fully capable of transmitting the disease.

Consider also the relative risk of the provoked bite of an apparently healthy dog in the following geographical areas:

a. New York City

b. rural Colorado (all rabies is rare in Colorado)

c. rural Missouri (canine rabies occurs sporadically)

d. a tropical foreign country (canine rabies is common)

It can be seen that the relative risk of the bites varies greatly (the basis for "rabies-free areas"); the bite of a dog in rural Missouri or in a foreign country must be considered a significant risk until proven otherwise. (It is clear that the geographical area in which the bite occurs must also be considered; this discussion focuses primarily on the United States.) Is the rabies risk of a given bite zero, 1:1,000,000, 1:1,000 or higher? The answer is seldom either zero or absolute. However, most anxiety stricken parents of even a severely bitten child can deal with the situation and decide upon the level of risk that is tolerable to them if they are given straightforward facts and answers to their questions and credible professional opinion. When answers are unavailable, fuzzy, or conflicting, the anxiety levels escalate.

In summary, the options available in the handling of a biting dog or cat are limited. They are:

a. Do nothing - this is undesirable in the eyes of the bitee, but often is the position of those in charge of the bitor.

b. Confine the biting dog or cat for ten days from the date of the bite to observe for the appearance of diagnoseable illness (confinement is frequently accomplished with difficulty for a variety of reasons).

c. Kill it immediately to examine the brain. 


\section{How Can Bites Be Better Handled} Using New Technology?

Situation A: A dog bites a person and promptly escapes, never to be seen again. What better can be done?

The dog is gone so that no sampling can be done. However, the widespread knowledge that new technology is available will stimulate more lay persons, veterinarians, and physicians to seek out expert assistance. Advisors to these situations can use the best knowledge in behavioral and risk assessment. In other words, functioning laboratories will provide a focus to upgrade the entire advisory effort, even when laboratory services are inappropriate or impossible.

Occasionally, the person bitten has previously received antirabies treatment or vaccine; the blood of the person can be tested for antibodies to determine if adequate protection is already afforded from the previous immunization. In some cases, it is desirable to take a blood sample to determine the antibody level after the series of injections is completed (this is not routinely the case), to assure that the person has in fact responded to the vaccine. The increased use of advisory services, and the analysis of blood of certain persons exposed for antibodies should decrease the need for the use of antirabies vaccine.

Situation B: A dog bites a person, is clearly sick or acting abnormally, and is confined and available for examination.

The animal should be evaluated by a veterinarian immediately who should make a tentative diagnosis of the illness, securing appropriate samples for analysis. If there is a discreet, diagnosable illness involved or at least one that is clearly distinguishable from rabies, the probability of concurrent infection with rabies virus is remote, but not impossible. However, a positive response to treatment of that illness is incompatible with and lessens the likelihood of the animal being ill with the virus. If the animal has a blood antibody titer against rabies virus (the higher the titer the more reliable), the chance of it being infected by a previous exposure to rabies is remote (Koprowski et al. 1954; Dean et al. 1964; Cabasso 1965; Baer 1975; Fekadu and Shaddock 1984). A blood serum titer in response to rabies virus infection appears and rises during the clinical course of the illness (Hattwick and Gregg 1975; Anderson et al. 1984) and must be carefully interpreted along with the clinical condition of the animal. A reliable history of immunization would explain a high or very early titer and make rabies virus infection most unlikely. If the animal does not have a titer of significance, the decision of provocation or lack of provocation and further evaluation become important; if a negative titer converts to positive, or a low titer increases, rabies infection is established. 
If evidence of unprovoked biting, and neurological or paralytic illness exists, immediate examination of brain tissue or skin biopsy specimens is strongly indicated, and the need can be balanced by the nature and severity of the bite exposure with the desirability of salvaging the animal.

If the illness is not strongly suggestive of being rabies, examination of skin biopsy specimens is indicated. In one study (Blenden et al. 1983), 133 animals (including many dogs and cats) with naturally occurring, undiagnosed illness, were tested for rabies virus using skin biopsy examination. Sixty-eight of seventy animals (97 percent) that were ultimately proven to have rabies had positive skin biopsy examinations. All of the sixty-three animals that were proven not to have rabies had negative skin biopsy results. These results are signficant because the animals did produce potential exposure of humans to rabies. This study thus suggests that such examination has high but not absolute sensitivity, and can of course be repeated should that be appropriate, as the passage of time (during the course of rabies) will make the next examination more likely to be positive (Blenden et al. 1983). As rabies in dogs and cats is unusual, the biopsy test results are most likely to be negative; the negative result must be considered within the context of the entire situation, and not taken as the last word. Following a negative biopsy, the animal is still alive, and the bite situation may dictate further evaluation.

In addition to skin and blood, cerebrospinal fluid can also be examined. Analysis of cerebrospinal fluid for antirabies antibodies will help demonstrate the immunization status of the dog, and whether this antibody is present as the result of vaccination. Thus, the dog that is sick and has an uncertain history of vaccination can be tested. If an antibody is present in the blood and absent in the cerebrospinal fluid, the dog can tentatively be assumed to not have rabies, and is still alive for appropriate observation. If the blood contains no antibody, it does not mean the illness is rabies, merely that the dog is more likely to be susceptible to rabies. If antibody is present in cerebrospinal fluid, and not in the blood, present or past infection with rabies virus is suggested (Bell 1975). Anderson et al. (1984) state that blood serum and cerebrospinal fluid antibody appear at about the same time in their sampling of human cases.

\section{Situation C: A healthy-appearing dog bites a person, is captured,} and is known to be a friendly type of dog.

The basic question in this situation is whether the bite reflects normal and expected behavior of the animal, or is an act of unexplained aggression, symptomatic of rabies. If the animal has a significant blood antibody titer (as from immunization) it is most unlikely that rabies infection is involved, although still not impossible. If the blood is negative, the possibility of rabies infection is not ruled out, but a second blood sample would likely show a titer if rabies was involved. As the skin biopsy test is relatively accurate in detecting rabies in a sick individual, a positive result is mean- 
ingful and high sensitivity is expected if rabies encephelitis has progressed to the point of producing biting behavior.

An animal in this category, healthy-appearing, having inflicted a bite under unprovoked conditions, and with a negative blood and skin biopsy, must be further assessed for the possibility of being a presymptomatic carrier. Therefore, further clinical observation, another skin biopsy (no less than three days after the first), and a repeat blood antibody level are indicated; all, however, on a living animal.

Situation D: A healthy-appearing dog bites a person, is captured, and is known to be an aggressive dog or habitual biter.

On the assumption that the bite was normal behavior for the dog, the remaining risk is to determine the likelihood that the dog is a presymptomatic carrier (i.e., incubating the disease, possibly shedding virus in the saliva, and destined to develop rabies within a ten-day observation period). The finding of blood antibody indicates that the dog has resistance to rabies virus and is most unlikely to be incubating the disease; its bite is therefore not considered a rabies exposure. Assuming the dog was actively incubating rabies (and the bite was thus dangerous), there is an estimated 25 percent chance that the skin examination would be positive. That chance increases every day that passes from that time on. Therefore, a negative biopsy should be followed by observation and perhaps another biopsy in three to five days, especially if the serum antibody test is negative. Serum antibody will appear if infection is present.

\section{What Are The Strengths And Weaknesses of Application of These Methods?}

The addition of specific technology of defined sensitivity to detect the presence of rabies virus infection can add significant confidence to existing methods of evaluation. It is quite feasible that observation periods can be reduced in many cases, although they cannot be eliminated. Conversely, it may on occasion be desirable to confine a few days longer than ten (perhaps fourteen), particularly dogs having had rabies exposure outside the United States (Fekadu et.al. 1982). The killing of dogs and cats to examine brain tissue can be reduced to only the most essential cases. The overall quality of risk assessments of bite cases should markedly improve, reducing the need for antirabies treatments in many cases; this will occur simply as medical professionals better realize that alternatives are available. As the technology is highly specialized, the services can be available in relatively few laboratories in the United States. The shipment of specimens unfortunately requires more time than the laboratory examinations. A distinct weakness of skin 
biopsy technology is that early clinical cases of rabies are detected at perhaps a 50 to 90 percent sensitivity level when 100 percent sensitivity is desirable; however, occasional cases are positive even before the onset of symptoms. Sensivity of the testing is adequate, however, to justify application as long as the results obtained are used in the proper context of the entire clinical situation. As is true with most laboratory tests for diagnosis of disease, complete reliance on a single technique is seldom justified. Some costs involved in confinement and antirabies treatments will be reduced, however, overall costs will not be reduced because of surgical and laboratory fees.

An important question in evaluating dog and cat bites relates to the immunization status of the animal. While rabies vaccine, when properly administered, is highly effective, it is not absolute in protective capability (as is true with any vaccine). Some animals inherently do not have the ability to respond adequately, and this fact cannot be known without highly specific testing. Also, improperly handled or administered vaccine loses immunogenicity. The properly immunized dog or cat has a minimal chance of contracting rabies if exposed. Exposure to a large dose of virus (as by the bite of a rabid skunk) can override the immunity, particularly if it is waning due to the passage of time. If the immunization history is inadequate or unreliable, it will not help the evaluation of rabies risk of a bite.

The presence of an anti-rabies antibody titer in the blood reveals much about the resistance of the animal to natural infection. Most properly immunized animals will respond with a blood titer, although a few may not. However, it is important to realize that those that do not respond with a titer probably have resistance to the infection anyway because of cellular forms of immunity not detectable by examination of blood serum. Conversely, an occasional animal with an antirabies titer can be infected with rabies virus (Dean et al. 1964) due to a large dose of virus in the exposure or inadequate cellular response within the animal. Very large challenge doses of virus probably are rather common in artificially induced rabies, and are not as likely to occur in nature.

An additional use of serological testing in evaluating the immune status of an animal is to administer one dose of rabies vaccine and secure a blood antibody titer two to three days afterwards. If the animal has been previously immunized, there should be a significant (and rapidly rising) antibody titer at that time. An animal that responds in a positive manner to this test, has minimal chance of being actively infected with rabies virus. 


\section{The Procedures Involved}

Specimens acquired from the animal under test are relatively simple to obtain. Circulating antibody is measured in the blood; blood is easily obtained in small quantity for the purpose. To secure cerebrospinal fluid to measure antibody which is useful in determining whether antibody has been produced by infection or immunization, requires light surgical anesthesia, clipping and disinfecting a small area of skin at the base of the skull (back of the neck) and insertion of a needle into the spinal canal at that point. Only one to two milliliters of fluid are required. The comparison of antibody titers in the blood and cerebrospinal fluid are presented in Table 1 . These antibody levels are guidelines only and must be interpreted by a veterinarian experienced in their use; "low" and "high" are relative terms needing supporting information.

Table 1. Neutralizing antibody levels in blood serum and cerebrospinal fluid as a result of clinical rabies or immunization against rabies virus: Guidelines for interpretation.

\begin{tabular}{|c|c|c|c|}
\hline & & Antibody Level & \\
\hline Status & Blood & Cerebrospinal Fluid & Change \\
\hline Immunization & Negative to high & Negative or low* & Stable \\
\hline Infection & $\begin{array}{l}\text { Negative early: } \\
\text { then low to high }\end{array}$ & $\begin{array}{l}\text { Negative early; } \\
\text { then low to high }\end{array}$ & Increases \\
\hline
\end{tabular}

*The physiologic ratio of antibody between cerebrospinal fluid and blood is 6:100 (Adapted from Bell 1975; Hattwick and Gregg 1975).

The biopsy of skin tissue to examine under the microscope is a minor surgical procedure. From the dog, the ideal anatomical site is known as the lateral cheek papilla, a small raised area on the cheek (one or two on each side), having three to four tactile hairs or "whiskers" growing from each. The follicles of these tactile hairs, in the deeper layers of the skin, are surrounded by a complex of nerve fibers, and it is in these nerve fibers that the rabies virus is found in the infected animal. The dog is given a light surgical anesthesia, or local anesthetic is carefully infiltrated around (not into) the area, the skin clipped and disinfected, and a small (1/4" diameter) plug of skin removed, making sure that the follicles of tactile hairs are included. A special biopsy punch is used for this purpose. The hole in skin is usually not sutured, merely kept clean with antispetics, and heals rapidly. Should any unusual type of infection develop, the wound requires further veterinary attention. 
The tissue specimen is placed into a small specimen container, protecting it from drying, and refrigerated or frozen for delivery to the laboratory, the specimen must be packaged and shipped refrigerated or in dry ice in a molded styrofoam shipping container, sufficient to maintain the tissue cold or frozen until delivery to the laboratory; the best taken specimen is worthless if it partially decomposes en route. The day seems close when skin specimens can be sent in $10 \%$ formalin, not requiring refrigeration at all, but, as of today, there is no substitute for refrigeration; formalinized brain or other nerve tissue can be accurately processed now (figure $1,2,3$ ).

The specimen is cut into ultrathin sections $(10 \mathrm{~nm})$, stained with special reagents (for immunofluorescence or immunoperoxidase staining) which react specifically for rabies virus, and are then examined under an ordinary or ultraviolet microscope, depending upon the procedure being used. In rabies, the virus gathers together in small clusters that are visible microscopically.

There are other tissues which can be utilized as well, in exceptional circumstances, in order to evaluate a specific case. For example, smear

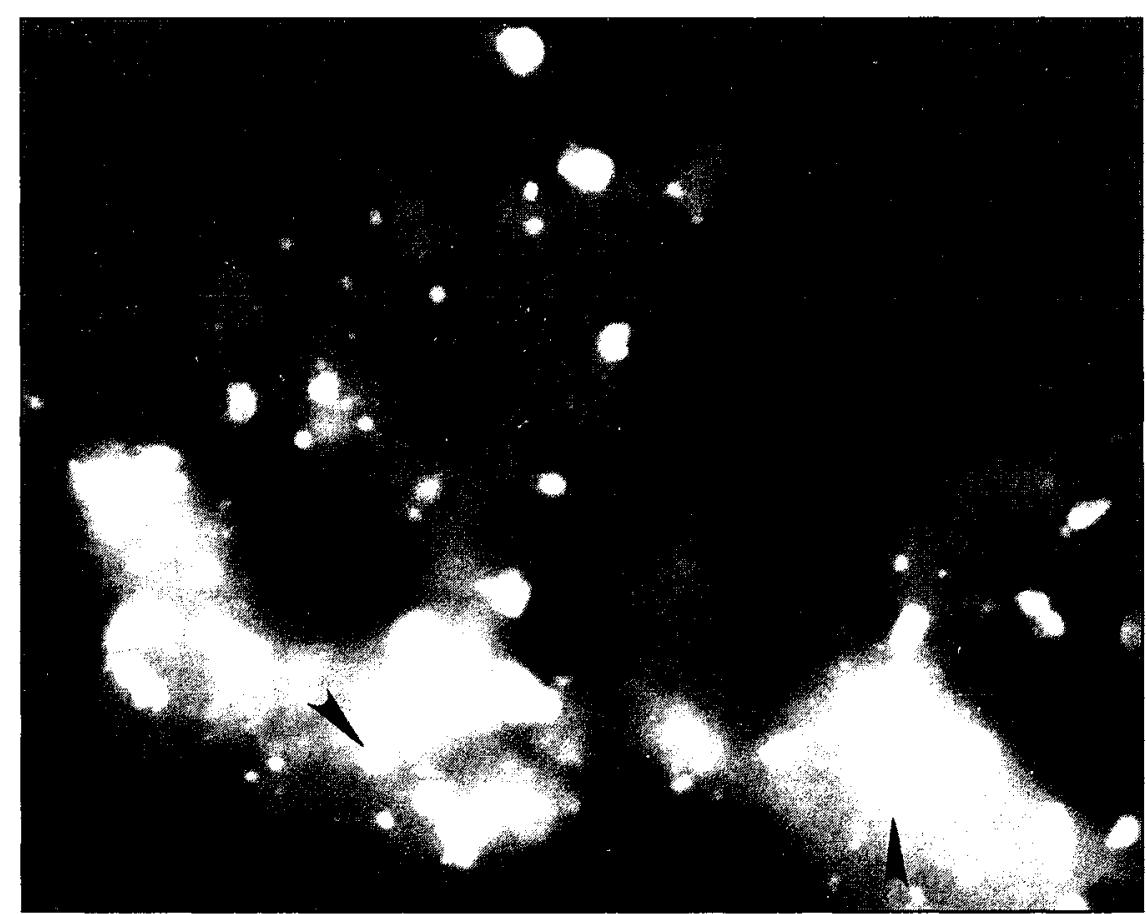

Figure 1: Fox cerebellum with natural rabies infection; immunofluorescent antirabies staining of tissue prepared by frozen sections. The tissue was stored in $10 \%$ formalin for three years and was not treated with trypsin. Magnification $1020 \mathrm{X}$ oil immersion, using ultraviolet illumination. 


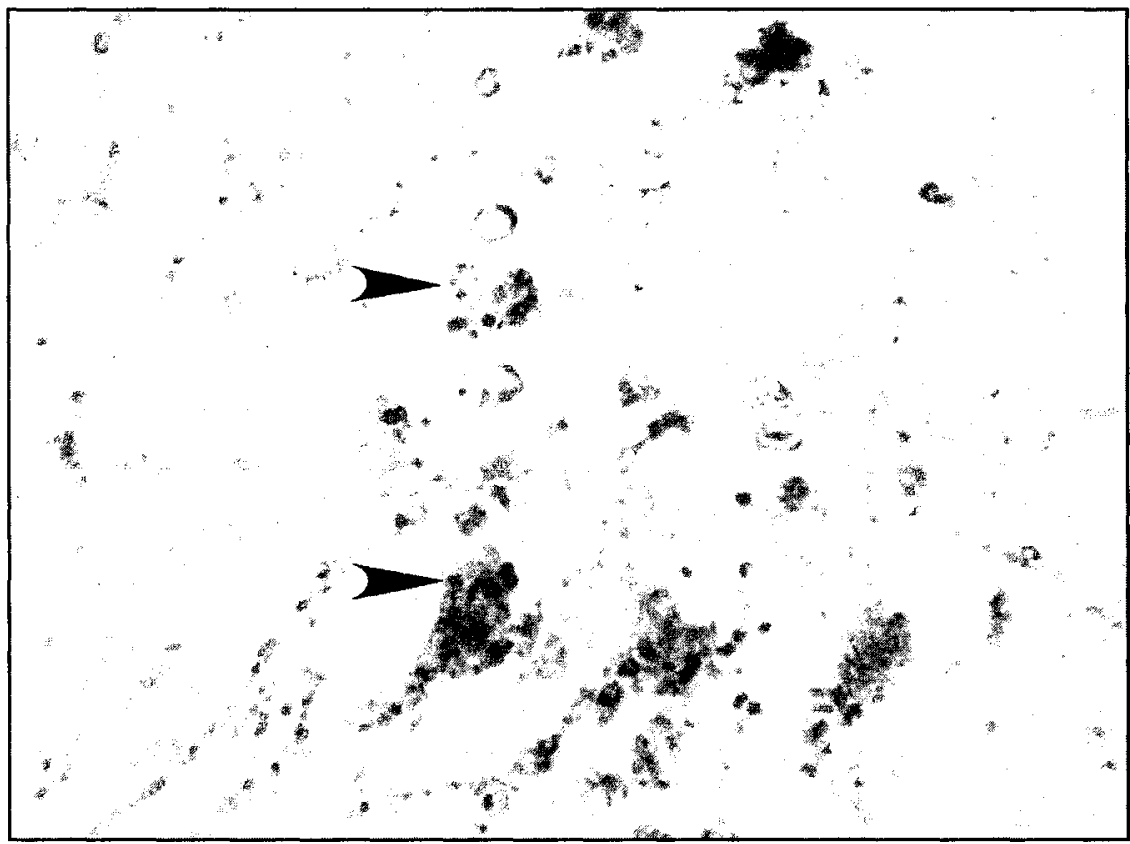

Figure 2. Fox cerebellum with natural rabies infection; immunoperoxidase antirabies staining of tissue preserved in $10 \%$ formalin for three years before paraffin embedding. The tissue was not treated with trypsin. Magnification 1020X oil immersion, using incandescent illumination.

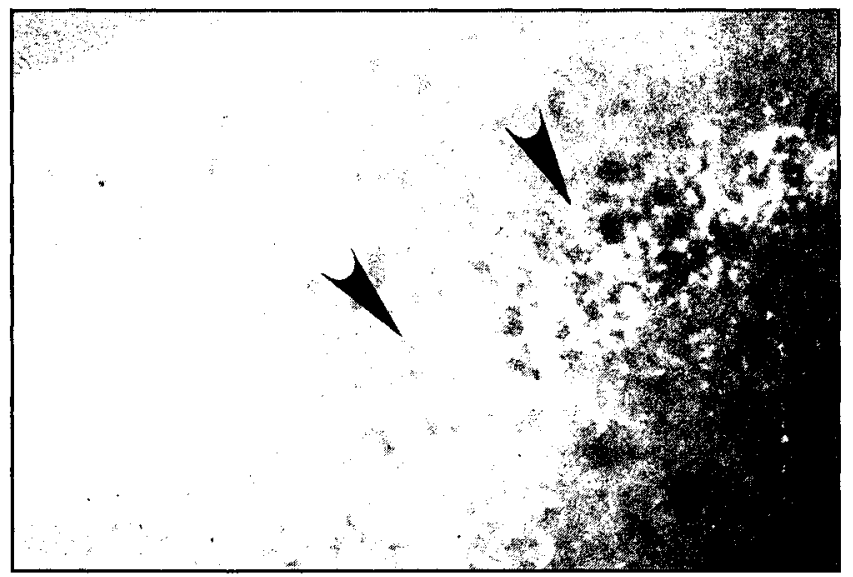

Figure 3. Mouse hippocampus infected with CVS-11; immunofluorescent antirabies staining of formalin-fixed paraffin embedded tissue. The tissue was acetone fixed and treated with $0.025 \%$ trypsin. Magnification 200X, using ultraviolet illumination. 
preparations of the cornea can be carefully prepared and examined microscopically (Schneider 1975). This test seems comparable in sensitivity (when adequate numbers of cells are examined) to examining the nerves of the skin; it is quite specific when found to be positive. Acquiring the specimen correctly to have adequate cells to examine, and not damage the eye, is quite exacting. In exceptionally valuable animals it is also feasible to secure a biopsy specimen for microscopic examination from the brain itself by a neurosurgeon. Results obtained via this method also must be evaluated carefully, as the virus of rabies is not uniformly distributed throughout the brain tissue; the biopsy specimen secured may be just the area having little or no virus present (low or uncertain sensitivity; high specificity).

The rabies virus is widely distributed in other neural tissues close to the brain such as the cranial nerves (Umoh and Blenden 1982). These tissues can be examined when brain is unavailable, with high accuracy. Particularly suitable are the trigeminal nerve (gasserian ganglion), the optic nerve, and the ganglion cell layer of the eye, and the tongue (taste buds). Recent findings indicate these sensory nervous tissues are infected only centrifugally; thus the sampling of those sites for diagnosis increases the chances of success (Torres-Anjel et al. 1984a). Other cranial nerves also contain virus in the infected animal; it is wise to sample several of the cranial nerves as close to the brain as possible when brain is not available. The cervical and other portions of the spinal cord are also valuable tissues to examine, likely comparable to the brain (Fekadu and Shaddock 1984).

Especially useful is the ability to utilize tissues which have been fixed in formalin. This is accomplished by immunofluorescence using $0.25 \%$ trypsin digestion to decouple the polypeptide chains formed by the fixation and expose the virus antigen sites (Umoh and Blenden 1981). A trypsin and pepsin sequential digestion presumably works by the same mechanism (Reid et al. 1983). Improved results are obtained using only $0.025 \%$ trypsin (Torres-Anjel, M.J., unpublished data). We have had superior results without the use of enzymes using peroxidaseantiperoxidase staining on formalin fixed tissues which have been shipped internationally and stored for extended periods (Torres-Anjel et al. 1984a,b), (Figures 1, 2, 3).

The biopsy procedure is basically identical for the cat as the dog, except that the specimen must be taken from the muzzle, securing as many follicles of tactile hairs as possible. The wound resulting from the biopsy is slower to heal on the muzzle, as it easily becomes infected. Rapid healing of the wound requires scrupulous care with cleaning and antiseptics for a few days. 


\section{Sensitivity and Specificity of the Tests}

One of the most difficult propositions to be faced in laboratory diagnosis of disease, is the theoretical reality that a relative few sick individuals have to be differentiated from within a much bigger pool of individuals. Those individuals with the disease and showing symptoms are more likely to be test positive, those with the disease and not showing symptoms are less likely to be test positive (more likely to be test negative) and that those without the disease are even less likely to be test positive (and even more likely to be test negative). Naturally, "disease" and "test" must be carefully defined.

As biological phenomena are rarely absolute, there is always the probability (and presence) of false positive and false negative results. Even individuals with the same disease within the population do not display identical signs. The distribution of both individuals and test

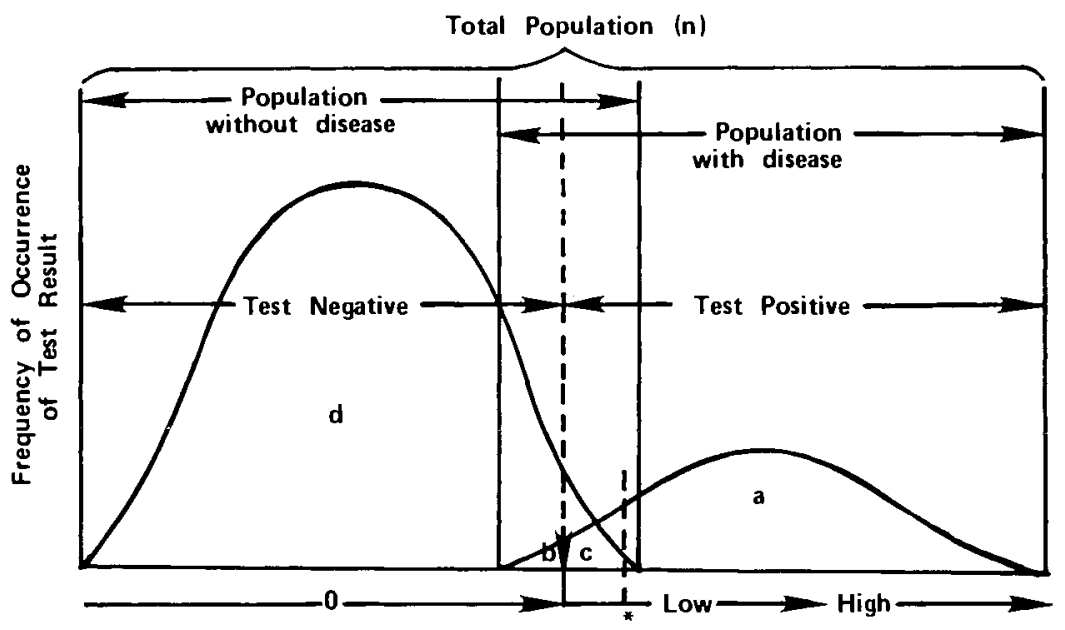

Test Units

*Test result threshhold of positive or negative is usually subjectively determined; depicted is estimated threshhold for skin biopsy testing for rabies (i.e., high specificity and lower sensitivity).

$a+b+c+d=n$

$a=$ with disease and test positive (true positive)

$b=$ with disease and test negative (false negative)

$c=$ without disease and test positive (fa/se positive)

$d=$ without disease and test negative (true negative)

Figure 4. Determination of sensitivity and specificity of reference laboratory tests on a population of individuals. 
results (in a population of adequate size) follows a typical sigmoid curve; problems producing confusion occur when the two sigmoid curves overlap. Referring to figure 4, curve "a" depicts those individuals that do have the disease (i.e., meet defined criteria); these are true positive test results. Curve " $\mathrm{d}$ " depicts those individuals that do not have the disease and are test negative (meeting defined criteria), representing the true negative test results. Subsegment "b" depicts those individuals that do have the disease and are test negative representing the false negative test results. Subsegment "c" depicts those individuals that do not have the disease and are test positive, comprising the false positive test results. In rabies testing, as the tests are highly specific, one expects to see a higher proportion of false negative than false positive tests.

Difficulties may be compounded when in reality we cannot even reach a theoretical truth with which to compare. This statistical approach assumes the calibration of a reference test against a true diagnosis. In practice, this model is much more complicated since one may be mostly calibrating a new (e.g., "field" or "screening", etc.) test against a reference test; the latter itself carries the statistical difficulties already mentioned and the new test will amplify the complications of sensitivity and specificity. There is not a perfect test, so that adequate management of imperfections is the clue to diagnostic decision-making. It is this ability on the part of the diagnostician that helps comprise the art of diagnostics.

This well-accepted concept of biomodal distribution has given rise to terminology and measurements of sensitivity and specificity as estimates of the accuracy and precision of a given test. Biologically, a test with a sensitivity and specificity of 1.0 (100 percent) does not exist; some examples may appear to do so, based on the particular sampling made available and observed from the universe of all true cases of the disease, including those cases not recognized. It is for the scientific, medical, and involved segments of our society to decide what degree of reliability (sensitivity and specificity) can be tolerated (table 2).

For example, in the laboratory diagnosis of rabies, the brain examination by fluorescent antibody microscopy is the recognized reference test, although not 100 percent sensitive and specific; extremely high to be sure, but not absolute. A problem immediately emerges as the tests for rabies have profound implications, so that anything less than 100 percent confidence and accuracy in interpretation escalates anxiety levels. Rabies diagnostic laboratories, when confronted with a test result that is not clear-cut, often report it as positive, as the results of the test will govern whether a person receives antirabies treatment or not. If error is involved, safety for the exposed individual must determine the result reported. The unwritten goal of routine diagnostic testing for rabies is to determine the need and appropriateness of treatment, rather 
Table 2. Calculation of sensitivity and specificity of laboratory tests on populations of individuals.

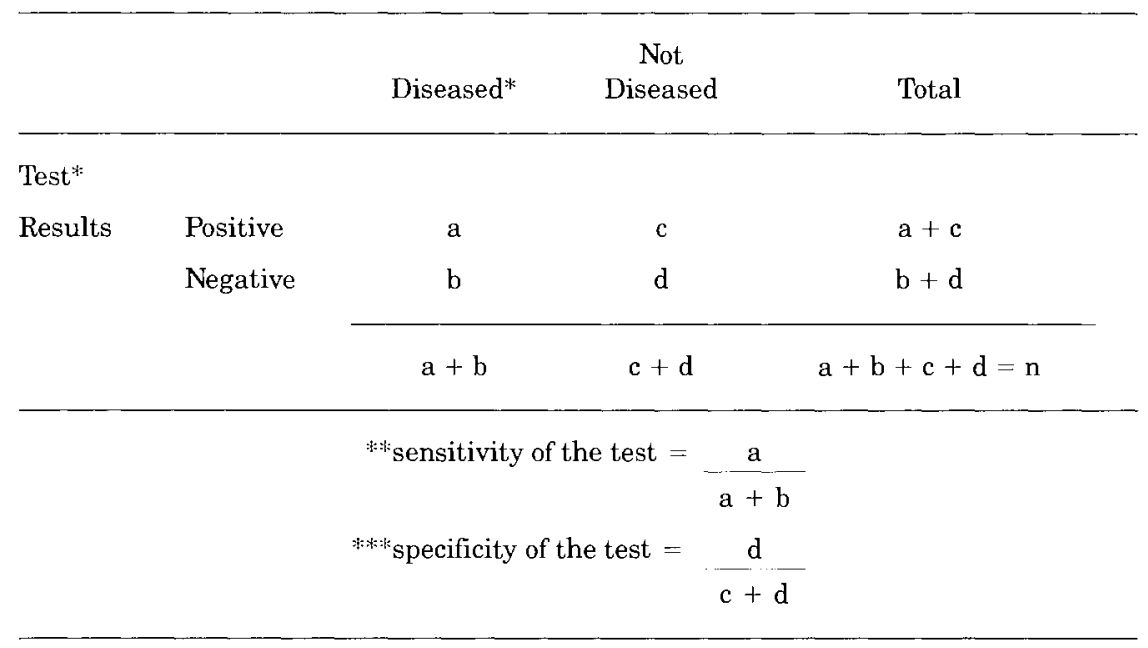

* "Test" and "diseased" must be specifically defined

$* *$ Ability to detect as positive those that have the disease $(1.0=100$ percent $)$

*** Ability to detect as negative those that do not have the disease $(1.0=100$ percent $)$

than to search for an absolute and accurate description of the presence or absence of infection by rabies virus.

An occasional case of rabies is not typical and requires additional effort and testing procedures to supplement the routine; additional time, high costs, and delay in treatment are involved, making this an unrealistic protocol for the routine diagnostic laboratory. Thus, the underlying goal of the testing (i.e., whether to treat or not) tempered by the realisms of time, money, and high specimen numbers in laboratories really determine the tolerable levels of specificity and sensitivity. Testing for diagnostic purposes has certain goals and limitations; testing for research purposes has a different set of goals and limitations. The search for 100 percent sensitivity and specificity even involves rather complicated statistics to assure significance (Buck and Gart 1966; Gart and Buck 1966).

Table 3 displays the calculated sensitivity and specificity of the examination of skin taken from various species and time-oriented situations. The commonly occuring figure of 1.0 in this table should not be construed to mean that the tests are perfect, which has been explained as unrealistic to expect; 1.0 should rather be considered as "close to 100 percent" as larger sample numbers would disclose an occasional discrepancy. Note also that specimens secured antemortem have lesser levels of sensitivity than those secured in terminal cases or postmortem; 
Table 3. Sensitivity and specificity of skin examination for detection of rabies virus antigen in nerve fibers of skin secured antemortem and postmortem

\begin{tabular}{|c|c|c|c|}
\hline Species & Total Observations & Sensitivity & Specificity \\
\hline & \multicolumn{3}{|c|}{ Antemortem Experience } \\
\hline $\begin{array}{l}\text { Dogs, natural infection, } \\
\text { all antemortem stages }\end{array}$ & 47 & .93 & $1.0^{*}$ \\
\hline $\begin{array}{l}\text { Dogs, experimental infection, } \\
\text { all antemortem stages }\end{array}$ & 46 & .68 or $0.0^{\text {*** }}$ & 1.0 \\
\hline $\begin{array}{l}\text { Dogs, experimental infection, } \\
\text { first day of onset }\end{array}$ & 46 & .55 & 1.0 \\
\hline Human, all antemortem stages & 20 & .60 & 1.0 \\
\hline \multirow{2}{*}{$\begin{array}{l}\text { Mixed animal species with } \\
\text { naturally occuring illness, } \\
\text { all antemortem stages }\end{array}$} & $136^{* * * * *}$ & .98 & 1.0 \\
\hline & \multicolumn{3}{|c|}{ Postmortem Experience } \\
\hline $\begin{array}{l}\text { Mixed species, natural and } \\
\text { experimental infections }\end{array}$ & 251 & .99 & 1.0 \\
\hline $\begin{array}{l}\text { Dogs, two separate } \\
\text { experimentally infected } \\
\text { groups only }\end{array}$ & 13 & 0.0 & 0.0 \\
\hline Goats, experimental infections & 38 & 0.1 & 1.0 \\
\hline
\end{tabular}

* All values of 1.0 should be interpreted as "close to 1.0."

*** Two separate experimental groups of dogs, inoculated with two viruses produced totally negative and unexplained results; these (13) are not included in the total observations. The extensive collaboration of Dr. J.F. Bell is acknowledged.

**** One young skunk, naturally infected, had positive skin two days before symptoms developed. 
the sensitivity on the day of onset of a series of experimentally infected dogs was .55 ( 55 percent), increasing through the course of the disease until it reaches 1.0 (100 percent) at the time of death (table 3$)$. This increase through time (the course of illness) has been observed previously in experimentally infected mice (Blenden 1983) and in human cases of rabies (Blenden 1978). It is natural that the most critical need in the in vivo assessment of the biting dog or cat is as a predictor of the onset of rabies, or early in the clinical course of the illness as those with advanced illness are usually obvious. These types of data are virtually impossible to secure from naturally occurring cases, especially the dog and cat. There is reason to feel, however, that the onset of cases of dogs and cats may be predicted with a low degree of sensitivity (estimated .25 or below). Such predictive value has been observed in experimentally infected mice (Blenden et al. 1983) and in a naturally occurring case in a skunk kitten (Blenden 1981). Anderson et al. (1984) have observed that about 50 percent of human cases (which occurred between 1960-1979) were detected early in their clinical course by neck skin biopsy (three of four cases) or corneal impressions (four of twelve cases), a figure that agrees with our own experience (skin biopsy) on a larger number of cases (Blenden et al., unpublished data).

Some results relating to the sensitivity biopsy testing are quite baffling. For example, experimentally infected goats have a very low sensitivity of skin biopsy in detecting rabies infection antemortem. The same has been observed in some experimentally infected dogs, usually when the incubation period is short (abnormally short when compared to naturally occurring disease) (Umoh and Blenden 1982; Fekadu and Shaddock 1984). There is also likely a difference in the patterns of invasion of different strains of virus, and their dissemination into nerves of the skin. Virus strains used experimentally are uniform and used in groups of animals; only limited numbers of virus strains (even though they are "street virus") can be used. Naturally occurring infections on the other hand, are produced by a large and heterogeneous selection of virus strains, each differing slightly from another in their ability to infect and disseminate. Fortunately, skin biopsy seems more reliable in naturally occurring infections than in those which are artificially induced. Unfortunately, it is virtually impossible to secure a number of naturally occurring cases very early in their clinical course (when the information is most valuable), and so these data must be secured by experimental means.

An algorithm is presented (figure 5) to graphically depict the criteria and decision points which have been discussed and which may be considered in evaluating the rabies risk of $d o g$ and cat bites inflicted on humans. The early part of the algorithm, covering the common happening wherein the biting animal is not available for examination, has been previously published by other authors (Corey and Hattwick 1975). 


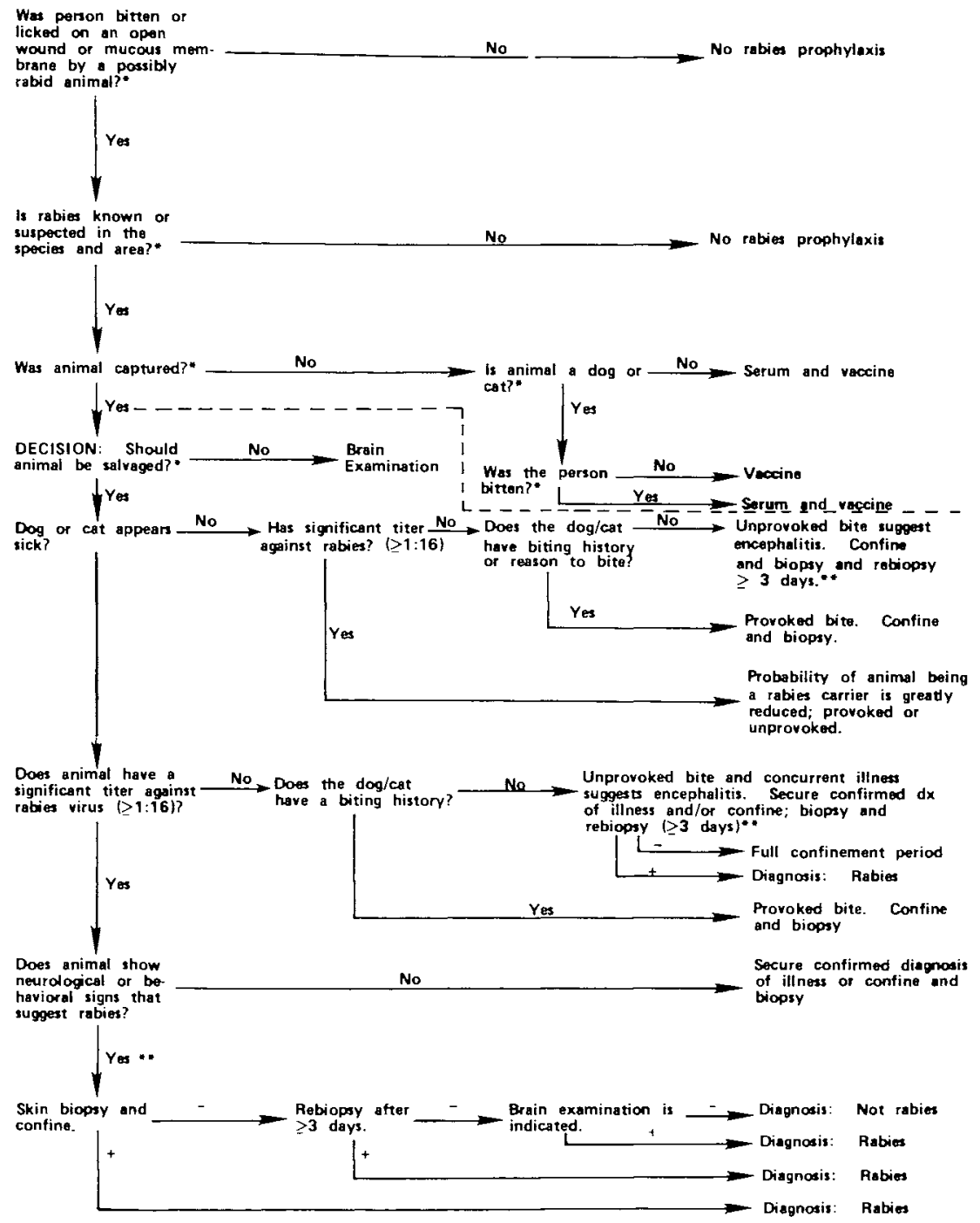

- Corey, Lawrence and Michael Hattwick. 1975. Treatment of Persons Exposed to Rabies.

Journal of the American Medical Association. 232: 273.

- Initiation of antirabies treatment should be considered pending observations.

Figure 5. Steps to consider in the evaluation of rabies risk from dog or cat bites inflicted on humans (algorithm). 
The remainder of the algorithm has been developed by blending traditional practices with new alternatives (in the form of laboratory examinations) into a decision tree. The decision pathways of the algorithm are constructed so that traditional means can be immediately resorted to if an unexpected event seems to warrant; it is likely that usage will suggest minor modification or refinement.

\section{Summary and Conclusions}

Dog and cat bites are increasing in importance to our society; dog bites especially are alarming in frequency and severity. Both species maintain a significant risk of rabies. Each bite represents a potential rabies risk requiring evaluation; some are slight or negligible in risk, other are high in risk. Concurrently, with the increase in dog bites, the relative risk of rabies exposure has decreased tremendously. In the 1950 's, we officially recognized thousands of cases of dog rabies per year in the United States; due to immunization and stray control, the figure is now a few hundred per year. The technology of current evaluation for rabies risk of dog and cat bites was developed prior to and in the early 1960's, consisting of confinement and observation and immunofluorescence examination of brain tissue. Antirabies treatments administered to people are documented to be excessive (in retrospect), suggesting that easier availability and more active use of advisory services can reduce markedly the number of antirabies treatments. The evaluation processes of confinement and observation or examination of brain tissue can be reduced by the application of supplementary resources: i.e., better use of advisory services and the use of certain laboratory procedures to supplement information to make bite evaluations more objective.

A valid objective to consider is that positive and objective attitudes can be applied to these bite evaluations in order to accommodate for new technology and additional parameters. Considering the greatly reduced risk of dog bites and carefully separating the low risk majority from the high risk minority, the killing of dogs or cats solely to examine brain tissue is seldom justified. Periods of post-biting confinement and observation can be shortened or perhaps eliminated in selected cases; extended periods may be appropriate in select situations.* One must be careful not to compromise the sensitivity of the evaluation process, as even with greatly reduced risk, the exceptional cases will occur somewhere, and the "impossible" sometimes happens. At any rate, the

*Four of nine dogs artificially infected with an Ethiopian origin virus had virus in saliva thirteen days before onset (Fekadu 1984). 
addition of new technology to the resources available can greatly reduce the killings for examination of brain tissue.

Laboratory technology which can complement existing methods centers around the detection of antirabies antibody both in the blood and cerebrospinal fluid (in order to differentiate antibody originating from infection versus immunization, and to establish the level of resistance of the animal) and the detection of virus antigen by examination of tissue available from the living animal (e.g. corneal impression smears and skin obtained by biopsy). The availability of alternative procedures will also focus on the easier availability of bite exposure counseling services to eliminate defensive and excessive antirabies treatments. The tremendous emotional distress which so often accompanies bite events can be greatly alleviated simply by developing more objective and accurate information about the degree of risk involved, and by having available laboratory technology to apply to the living animal, so that the common fear of brain examination can often be dispelled.

The sensitivity and specificity of laboratory tests, specifically as they apply to rabies are discussed. No test is perfect (even those in use today) so that reliance on a single laboratory test is seldom totally and absolutely justified. An algorithm is presented placing situations and actions regarding dog and cat bites into a decision tree.

Perhaps the biggest and most effective factor in the alleviation of this problem lies in the diligence used by owners with respect to their dogs and cats. It is well recognized that there is correlation between the level of responsibility assumed by dog and cat owners, and the bites that these animals inflict. If dogs and cats were properly and responsibly maintained, many less bites would occur, more dogs and cats would be immunized against rabies thus reducing the rabies risk of bites and the number of stray and unwanted dogs and cats would be reduced, resulting in less bites and a reduced reservoir of rabies.

\section{Acknowledgements}

The authors wish to gratefully thank B.N. Hamory, M.D. , J.F. Bell, M.D., Ph.D., R. Currier, D.V.M. and J. Martin, D.V.M. for review of portions of this manuscript and a continuum of advice and collaboration. 


\section{References}

Anderson, L.J., Nicholson, K.G., Tauxe, R.V. and Winkler, W.G. 1984. Human rabies in the United States, 1960 to 1979: Epidemiology, diagnosis and prevention. Ann. Int. Med. 100(5):728-35.

Beck, A.M. 1981. The epidemiology of animal bite. Compendium on Continuing Ed for Pract. Vet. 3(3):254-8.

Bell, J.F. 1975. Latency and abortive rabies. In: Baer, G. ed. The Natural History of Rabies, Vol. 1. New York: Academic Press.

Blenden, D.C. 1978. Identification of rabies virus antigen in the skin by immunofluorescent staining. Zoonoses (PAHO) I(1-2):7-11.

Blenden, D.C. 1981. Rabies in a litter of skunks predicted and diagnosed by skin biopsy. J.A.V.M.A. 179(8):789-91.

Blenden, D.C., Bell, J.F., Tsao, A.T. and Umoh, J.U. 1983. Immunofluorescent examination of the skin of rabies infected animals as a means of early detection of rabies virus antigen. J. Clin. Micro. 18(3):631-6.

Buck, A.A. and Gart, J.J. 1966. Comparison of a screening test and a reference test in epidemiologic studies. II. A probabilistic model for the comparison of diagnostic tests. Am. J. Epidem. 83:593-602.

Cabasso, V.J., Stebbins, M.R., Douglas, B.A. and Sharpless, G.R. 1965. Tissue culture rabies vaccine (Flury LEP) in dogs. Am. J. Vet. Res. 26:24-32.

Callaham, M. 1980. Dog bite wounds. J. Am. Med. Assoc. 244:2227-8.

Corey, L. and Hattwick, M.A.W. 1975. Treatment of persons exposed to rabies. J. Am. Med. Assoc. 232(3):272-6.

Dean, D.J., Evans, W.M. and Thompson, W.R. 1964. Studies on the Low Egg Passage Flury strain of modified live rabies virus produced in embryonating chicken eggs and tissue culture. Am. J. Vet. Res. 25:756-63.

Fekadu, M., Shaddock, J.H. and Baer, G. 1982. Excretion of rabies in the saliva of dogs. J. Inf. Dis. 145(5):715-9.

Fekadu, M. and Shaddock, J.H. 1984. Peripheral distribution of virus in dogs inoculated with two strains of rabies virus. Am. J. Vet. Res. 45(4):724-9.

Gart, J.J. and Buck, A.A. 1966. Comparison of a screening test and a reference test in epidemiologic studies. I. Indices of agreement and their relation to prevalence. Am. J. Epidem. 83:586-92.

Hattwick, M.A.W. and Gregg, M.B. 1975. The disease in man. In: Baer, G. ed. The Natural History of Rabies, Vol. II. New York: Academic Press.

Koprowski, H. and Black, J. 1954. Studies on chick embryo adapted rabies virus. V. Protection of animals with antiserum and living attenuated virus after exposure to street strain of rabies virus. J. Immunol. 72:85-93.

Marr, J.S. and Beck, A.M. 1976. Rabies in New York City, with new guidelines for prophylaxis. Bull. N.Y. Acad. Med. 52(5):605-16.

Reid, F.L., Hall, N.H., Smith, J.S. and Baer, G.M. 1983. Increased immunofluorescent staining of rabies infected formalin-fixed brain tissue after pepsin and trypsin digestion. J. Clin. Micro. 18:968-71.

Schneider, L.G. 1975. Spread of virus from the central nervous system. In: Baer, G. ed. The Natural History of Rabies, Vot I. New York: Academic Press.

Schnurrenberger, P.R, Martin, R.J., Merrdink, G.L. and Rose, N.J. 1969. Epidemiology of human exposure to rabid animals in Illinois. Pub. Hlth. Rpts. 84(12):1078-84.

Thomas, J.B. 1975. The serum neutralization, indirect flourescent antibody and rapid fluorescent focus inhibition tests. In: Baer, G. ed. The Natural History of Rabies, Vol. I. New York: Academic Press. 
Torres-Anjel, M.J., Montano-Hirose, J., Cazabon, E.P.I., Oakman, J.K. and Wiktor, T.J. 1984a. A new approach to the pathobiology of rabies virus as aided by immunoperoxidase staining. Amer. Assoc. Vet. Diag., 27th Annual Proceedings. pp. 1-26.

Torres-Anjel, M.J., Blenden, D.C., Oakman, J.K., Frost, J.W. and Satalowich, F.T. 1984b. International Symp. Med. Virology (Pan Am. Group on Rapid Viral Diagnosis). Dept. Pathology, Medical Center, Univ. of California, Irvine (In press).

Umoh, J.U. and Blenden, D.C. 1981. Immunofluorescent staining of rabies virus antigen in formalin-fixed tissue after treatment with trypsin. Bull. W.H.O. 59(5):737-44.

Umoh, J.U. and Blenden, D.C. 1982. The dissemination of rabies virus into cranial nerves and other tissues of experimentally infected goats and dogs and naturally infected skunks. Int. J. Zoonoses. 9:1-11.

U.S. Department of Health and Humane Services. 1983. Centers for Disease Control. Annual Summary 1980-82. Rabies Surveillance.

Vaughn, J.B., Gerhardt, P. and Paterson, J.L.S. 1963. Excretion of street rabies virus in saliva of cats. J.A.M.A. 184:705-8.

Vaughn, J.B., Gerhardt, P. and Newell, K.W. 1965. Excretion of street rabies in the saliva of dogs. J.A.M.A. 193:363-8. 